\title{
BMJ Open Approaches to governance of participant-led research: a qualitative case study
}

\author{
Azure Dominique Grant, ${ }^{1,2}$ Gary Isaac Wolf, ${ }^{2}$ Camille Nebeker $^{3}$
}

To cite: Grant AD, Wolf Gl, Nebeker C. Approaches to governance of participantled research: a qualitative case study. BMJ Open 2019;9:e025633. doi:10.1136/ bmjopen-2018-025633

- Prepublication history and additional material for this paper are available online. To view these files, please visit the journal online (http://dx.doi. org/10.1136/bmjopen-2018025633).

Received 6 August 2018 Revised 18 December 2018 Accepted 18 February 2019

Check for updates

(C) Author(s) (or their employer(s)) 2019. Re-use permitted under CC BY-NC. No commercial re-use. See rights and permissions. Published by BMJ.

${ }^{1}$ Neuroscience, University of California, Berkeley, Berkeley, California, USA

${ }^{2}$ Quantified Self Labs, Berkeley, California, USA

${ }^{3}$ Family Medicine and Public Health, School of Medicine, University of California, San Diego, La Jolla, California, USA

Correspondence to

Dr Camille Nebeker;

nebeker@eng.ucsd.edu

\section{ABSTRACT}

Objectives Participant-led research (PLR) is a rapidly developing form of citizen science in which individuals can create personal and generalisable knowledge. Although PLR lacks a formal framework for ethical review, participants should not be excused from considering the ethical implications of their work. Therefore, a PLR cohort consisting of 24 self-trackers aimed to: (1) substitute research ethics board procedures with engagement in ethical reflection before and throughout the study and (2) draft principles to encourage further development of the governance and ethical review of PLR.

Methods A qualitative case study method was used to analyse the ethical reflection process. Participants discussed study risks, risk management strategies and benefits pre-project, during a series of weekly webinars, via individual meetings with the participant-organisers, and during semi-structured interviews at project completion. Themes arising from discussions and interviews were used to draft prospective principles to guide PLR. Results Data control, aggregation and identifiability were the most common risks identified. These were addressed by a commitment to transparency among all participants and by establishing participant control via self-collection and self-management of data. Group discussions and resources (eg, assistance with experimental design and data analysis) were the most commonly referenced benefits of participation. Additional benefits included greater understanding of one's physiology and greater ability to structure an experiment. Nine principles were constructed to encourage further development of ethical PLR practices. All participants expressed interest in participating in future PLR.

Conclusions Projects involving a small number of participants can sustain engagement in ethical reflection among participants and participant-organisers. PLR that prioritises transparency, participant control of data and ongoing risk-to-benefit evaluation is compatible with the principles that underlie traditional ethical review of health research, while being appropriate for a context in which citizen scientists play the central role.

\section{INTRODUCTION}

This paper explores a case of participant-led research (PLR), which is defined as:

An activity that characteristically aims at the socially valued goal of producing

\section{Strengths and limitations of this study}

- A case study is traditionally considered a qualitative strategy to study a programme, activity or process in-depth, permitting description of processes or groups' responses to interventions in real-life contexts, like the one presented here.

- Qualitative case studies also have the benefit of deeply analysing a single unit with the intention of understanding how future iterations of similar units may function. We applied this methodology to study a participant-led research (PLR) project and anticipate that our results may contribute to the development of governance structures and ethical frameworks for future PLR.

- The discussions and interviews that comprised the data set for this manuscript occurred naturally as part of our ethical review process, and therefore did not introduce additional burden to participants.

- This study is limited by the composition of this relatively small and self-selected group; it was not designed to balance sex, age, educational background nor socioeconomic status.

- Although the authors have attempted to generalise across Institutional Review Boards/Research Ethics Committees whenever possible, the introduction to this study focuses primarily on research regulation in the US. As several participants in the case are from the European Union, this is a limitation.

generalisable health knowledge... It is distinctive as being initiated and conducted by the participants themselves. PLR includes individuals interested in acquiring health information, whether about themselves or more generally. ${ }^{1}$

This practice builds on over a decade of initiatives supporting public participation in the research process. ${ }^{2-5}$ Members of these initiatives, including community health workers, ${ }^{6}$ crowdsourced researchers ${ }^{4}$ and 'bio-citizens', ${ }^{7}$ align to the mutually beneficial goal of increasing the participation of everyday individuals in science. ${ }^{8}$ For example, in community-based participatory research ${ }^{9}$ and patient-centred outcomes research, ${ }^{10}$ 
community members or patients, respectively, work with professional scientists to shape the research questions most relevant to those participants. Relatedly, on citizen science platforms like Zooniverse ${ }^{511} 12$ and Citizen Science Alliance, ${ }^{13}$ individuals may contribute to hypothesis development, study design, data collection, data analysis or dissemination of results, while enabling greater scale and reducing costs for researchers. ${ }^{314-18}$ PLR combines characteristics of these initiatives in that it facilitates participant direction of all parts of the research process. ${ }^{19}$ Common reasons for engaging in PLR include: improving one's health via self-observation, ${ }^{20}$ gaining knowledge and support from others dealing with a common health condition $^{21}$ and contributing to the creation of useful tools. ${ }^{22}$ Despite its potential to contribute to the scientific literature, PLR publication is infrequent, even within the family of citizen science. ${ }^{152}$

A significant challenge to extending the impact of PLR is that research led by participants presents challenges to traditional methods of ethical review. ${ }^{924-27}$ Indeed, existing methods for ethical review may not be well-suited to the new challenges introduced by participant-led initiatives. ${ }^{26}{ }^{28-30}$ For instance, in PLR, the involvement of an academic institution may be peripheral or entirely absent. ${ }^{1931}$ Normally, the ethical and regulatory dimensions of scientific research are addressed by a regulatory body (ie, the Institutional Review Board (IRB) in the US; the Research Ethics Committee (REC) in the UK and $\mathrm{EU})$, whose role is to ensure that study risks are identified and managed, that benefits are appropriate in relation to risks, and that people are given the information needed to provide informed consent to volunteer. ${ }^{32}$ Although this review process was developed to protect research participants, the IRB (US), as its name clearly states, is an institutional process that was developed for use in an academic research context.

Given the novelty of PLR and the well-known history of harm caused by unethical experimentation in science, the participant-organisers of a PLR conducted in a selftracking community believed it was necessary to develop a process for ethical evaluation of their research plan, and to document procedures so that they could be critically reviewed and, if proven useful, be replicated or extended. Although an IRB was not utilised, participant-organisers were able to take advantage of the fact that IRBs/RECs have been well-described in an extensive literature on research ethics.

One such example is a guidance manual that was published in 2011 by the WHO. ${ }^{33}$ Because ethical review and oversight for research involving human participants can vary globally, the WHO developed this manual to standardise guidelines such that research taking place internationally would share expectations of competencies needed by a research ethics committee. ${ }^{34}$ The key criteria articulated in this document are the following: research must be designed in accordance with valid scientific methods; risks are minimised to the extent that they are reasonable in relation to the possible benefits; participants represent those most likely to gain from resulting knowledge; conflicts of interest have been evaluated; participant privacy and data confidentiality have been carefully considered; respect for persons is demonstrated via an informed consent process and the greater community is actively involved in the design and conduct of the research. ${ }^{34}$ Our ethical review process aimed to satisfy the high-level global requirements outlined by the $\mathrm{WHO}^{33}$ in a situation where the formal procedures of an IRB/REC were not applied.

\section{Case: ethical reflection in the blood testers project}

The idea for a PLR, 'Blood Testers', in which participants would frequently measure their own blood lipid levels, emerged from discussions at Quantified Self Meetups and conferences. Quantified Self $(\mathrm{QS})^{20}$ is a global community united by an interest in what can be learnt from self-collected data. Those affiliated with the QS community may be researchers, engineers or technologists, but formal research training is not required - only an interest in selftracking. Quantified Self Labs, a California-based limited liability corporation, provides administrative support, logistics and project leadership to the community. Quantified Self does not have an academic affiliation nor does it receive government funding to support research.

Project equipment was lent to participants by Quantified Self Labs; guidance on methods for ethical review was provided by participant $\mathrm{CN}$, a research ethicist. Additionally, several participants with prior academic research training agreed to share expertise. Although this was a group activity with a general, collective goal of learning about natural variability in blood lipid levels, each participant also developed an individual research question. That is, each participant in Blood Testers conducted a single-subject experiment based on an hypothesis of personal interest related to cholesterol and triglycerides. All participants and participant-organisers subsequently collected and analysed their own blood as often as once per hour using a commercially available blood lipid testing system.

Participants engaged in active discussion of risks and benefits of participation throughout the project. Participants and participant-organisers met to identify study risks and benefits; discuss what constitutes responsible conduct of PLR, including what information is needed to inform willingness to volunteer and to engage with media created for the project. At the project's conclusion, participants were interviewed about their experience in order to carefully assess perception of the project's ethical review process, allow participants to make final suggestions for improvement and to record any additional risks and benefits of participation. A step-by-step description of the process follows:

\section{Recruitment}

People affiliated with the QS community were provided with information about the project either through direct contact with the participant-organisers or via a session at the Quantified Self 2017 Global Conference. An example of information conveyed during recruitment follows: 


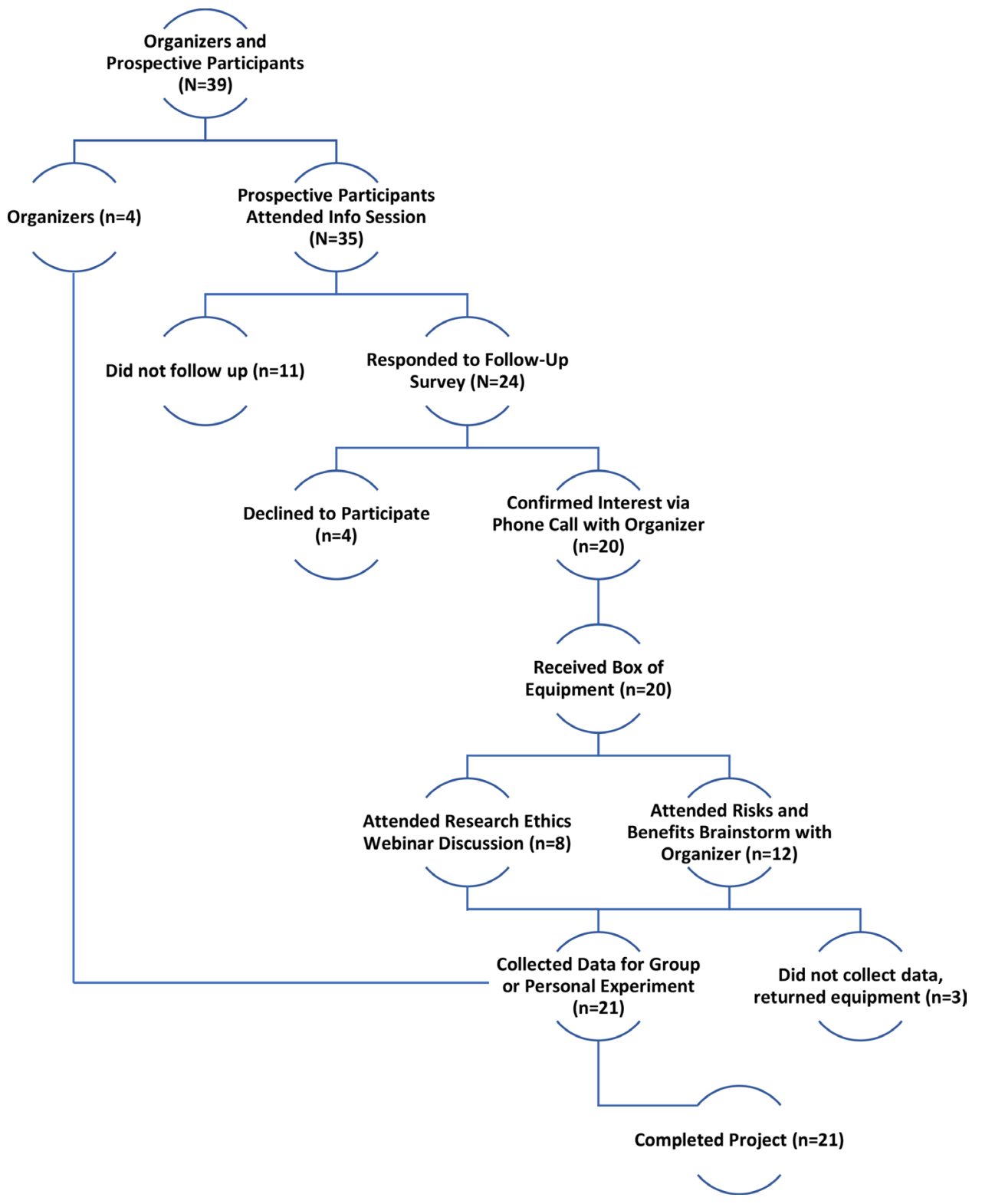

Figure 1 Phase Two. Recruitment Flowchart for the Quantified Self 'Blood Testers' participant-led research project: Four employees of Quantified Self Labs and 35 prospective participants met at the Quantified Self 2017 Global Conference to propose and discuss the project. Emails were collected and follow-up surveys were sent to gauge interest. Responders confirmed their interest in participation and their goal for the project with an organiser from QS Labs. These individuals received equipment and subsequently attended online discussions to brainstorm risks and benefits of participation. In total, 21 participants completed the project. QS, Quantified Self.

Cardiovascular disease (CVD) is the number one killer in the world. CVD risk is commonly assessed via annual point measurement of blood cholesterol and triglycerides. However, there is evidence to suggest that these outputs can vary significantly on short timescales. The Blood Testers project will explore whether collaborative self-tracking of cholesterol and triglycerides using a finger-prick assay leads to actionable, personal knowledge.

Following the session, potential participants communicated their interest via response to a survey and confirmed their intent to participate via email or phone call with the participant-organisers. Participants were then sent experimental equipment (figure 1 Recruitment).

\section{Training and data integrity}

All participants were trained to conduct a finger-prick lipid assay with the Clinical Laboratory Improvement Amendments, CLIA-waived CardioChek Plus (PTS Diagnostics, Indianapolis, Indiana) according to manufacturer's instructions. Training was delivered by participant-organiser AG via: (1) video tutorial, (2) live-webcast tutorial, (3) one-on-one Skype coaching or (4) in-person training. Each participant had access to one-on-one conversations with a participant-organiser throughout the 
project for any further training needed. Training efficacy was assessed first by the participant meeting or exceeding manufacturer's standards for accuracy and precision of cholesterol and triglyceride levels in a set of test samples. Training was considered complete if the participant met these standards and verbally expressed readiness to move on to experimental data collection.

\section{METHODS}

\section{Patient and public involvement}

As described below, participants co-led the recruitment, development and execution of this project. As one of the main goals of the study was to crowdsource participants' opinions and experiences to generate a list of risks and benefits, and another was to have each participant conduct their experiments based on personally-relevant questions, participant leadership was central. The study was designed with many available channels of communication for participants to exchange ideas before and throughout the study, as well as structured group discussions through which to do so. All participants were given the opportunity to read and comment on this manuscript prior to its publication. This manuscript will be disseminated open access such that all participants can view and share the work.

\section{Researcher characteristics}

In PLR, the 'researchers' also participate in data collection, and the 'citizen' participants also take on research duties (eg, contribute to ideas for data analysis, share software). Although only the authors met the standards for academic authorship, nearly all participants took on some type of organisational role or shared expertise, as described further below. In this manuscript, we refer to the authors/researchers as 'participant-organisers' rather than researchers, and acknowledge that although these individuals oversaw the project, participants acted as co-researchers. The authors were a leader in the Quantified Self community, GW, the leader of the project, $A G$, and the ethical review adviser/participant in the project, $\mathrm{CN}$. These individuals have past academic research experience in physiology and data science (AG), history and single-subject research (GW) and research ethics (CN). In this case, the authors were acquainted with most of those who decided to join the PLR through prior meeting at Quantified Self conferences. The fact that the participant-organisers were integrated into the community that conducted the project, and that several of the participants were long time community members, undoubtedly added some familiarity and ease to the project that would not have otherwise existed.

\section{Phase zero: blood testers pilot}

Prior to the beginning of the Blood Tester project, a pilot phase was conducted during which lipid measurement instrumentation was evaluated, equipment was purchased and potential research protocols were piloted. The group involved also initiated communications on a Slack, a project communication platform, to share questions, protocol drafts and updates on equipment selection and use. In preparation for the ethical review process, a research ethicist $(\mathrm{CN})$ known by GW was invited to join the group as a participant.

\section{Phase one: pre-participation ethical reflection}

At the official commencement of the project, a webinar was held during which participant-organisers and 11 prospective participants generated a list of risks, risk mitigation strategies and potential benefits of participation. A presentation by the participating research ethicist, $\mathrm{CN}$, summarised the principles of ethical research, including autonomy, beneficence and justice, ${ }^{35}$ with an emphasis on the purpose of informed consent. This session was repeated so that those who were not able to attend the initial session could contribute. Webinar training sessions were recorded and transcribed to maintain a running list of potential risks, benefits and attendance. Video recordings of webinar meetings remained available as a reference for participants throughout the project.

\section{Phase two: engagement via online and in-person group sessions}

As this project took place across six countries, and among participants from diverse educational and occupational backgrounds, it was decided that participants would be most likely to reflect seriously on the risks and benefits of participation if given multiple opportunities, described below, to do so.

\section{One-on-one meetings}

If participants were unable to join a group meeting, then a one-on-one meeting was scheduled with a participant-organiser. The same material was covered in these meetings, and any new risks or benefits uncovered were recorded. These sessions were continued or repeated as requested by participants and required an approximate total of 20 hours of conversation throughout the project.

\section{Written and video materials}

Discussions were summarised in a blog post to quantifiedself.com. A brief literature review providing background on the project was also available to participants on a shared Google drive and Slack channel. Based on participants' most common questions, two educational animations ${ }^{36}$ were created by AG to explain concepts in lipid physiology and biological time series.

\section{Data management}

Lipid data collected by each participant was controlled by that participant at all times. Participants could document their data privately on personal computers or notebooks or publicly via upload to a group Google sheet. Alternately, some participants opted to share their data privately with $\mathrm{AG}$, who led data analysis, without sharing publicly. At the conclusion of the project, data were 
removed from the public Google sheet unless participants explicitly asked to keep it online. Similarly, participants could opt-in to have their data de-identified and aggregated as part of a scientific manuscript. The manuscript was circulated prior to submission such that all participants could see how their data were represented and give feedback.

\section{Phase three: semi-structured interviews}

At the culmination of the data-collection period, semi-structured interviews were conducted with all finishing participants $(n=18)$, excluding the authors and non-finishing participants. The primary goal of the interviews was to better understand participants' risk and benefit evaluation, what factors they considered important for ethical review in PLR and what elements would be most useful to them in future PLR. For the complete list of interview questions see online supplementary table 1. Interviews took place over private webcast or phone and were recorded and transcribed verbatim. Participants were introduced to the purpose of the interview and were asked for permission to audio record the conversation. The interview protocol was developed by $\mathrm{CN}$, GW and AG, and AG conducted all interviews. As is common with qualitative methods, participants were encouraged to speak freely and not prohibited from sharing additional anecdotes about their experience with the project.

\section{Research design and analysis}

A case study methodology was chosen to examine the ethical review process of this PLR. The case study method is a form of empirical inquiry that can be used to study real-life phenomena (eg, decisions, programmes, implementation process, organisational change, etc) at an individual or group level. ${ }^{38-40}$ The case study method allows for a holistic investigation of group behaviour and processes and is useful in describing an intervention in real-life context, in this case the substitution of typical IRB procedures with a discussion-based ethical review process. Data collected specifically during dedicated discussions in phases one and three were analysed using content analysis techniques commonly used in qualitative research. ${ }^{41}$ The notes taken during the initial discussion focusing on ethical research practices as well as transcripts containing responses to the semi-structured interview questions were read line-by-line and then coded to identify themes and patterns independently by $\mathrm{CN}$ and AG. On completion of independent review, the researchers discussed themes and patterns and any disagreements in observations about the data were discussed until agreement was reached. Lipid data analysis for individual projects that occurred within the phase two period is not a focus of this paper and is not reported here. (figure 2: Timeline)

\section{RESULTS}

\section{Participant demographics}

The final group consisted of 24 participants, six women and 18 men, ages 22 to 70 years (median 36 years, SD 12 years) and 21 out of $24(88 \%)$ of participants completed the project. Participants lived in six countries: The US, The Netherlands, Denmark, England, Ireland and Austria; and were of white European, Middle Eastern, or Indian descent. Sixty-one percent of interviewed participants had no formal research experience, $23 \%$ had professional (eg, master's degree or higher in a scientific field) training, but were not career researchers, and $14 \%$ were actively pursuing a research career.

\section{Phase one}

A total of 11 participants contributed to the initial discussion about ethical dimensions of the project. See table 1 , box 1 for brainstormed risks and risk mitigation strategies and benefits, respectively.

\section{Phase two}

Documentation and results of this phase have been submitted separately for publication. ${ }^{42}$

\section{Participant hypotheses}

Topics of investigation included daily rhythms in lipids, cholesterol fluctuation across the menstrual cycle and the effects of switching to a plant-based diet on within-a-day and across-days variability of cholesterol and triglycerides.

\section{Reflections on ethical dimensions: risks and benefits of participation}

Risks regarding data management, including sharing of their personal health data, and privacy expectations dominated participant responses. Even participants who were willing to share their data in this project expressed that privacy was a main concern that would need to be addressed as PLR expanded. No participant proposed that the project posed a risk to their physical well-being. Although the risk of infection from finger-prick device and risk of pain from testing were raised as hypothetical concerns, they were rejected by all participants as negligible.

Using transparency to mitigate risk in participant-led research When talking about how to reduce risks, participants referred frequently to 'transparency' regarding the nature of the sponsorship for the project; how data are stored, aggregated and shared and data ownership. Maintaining transparency via frequent communication thus became a key principle that helped build trust between participants and participant-organisers. Communication occurred through several formats, including group webinars, one-on-one meetings with a participant-organiser, Slack chat window and written/ video material. 


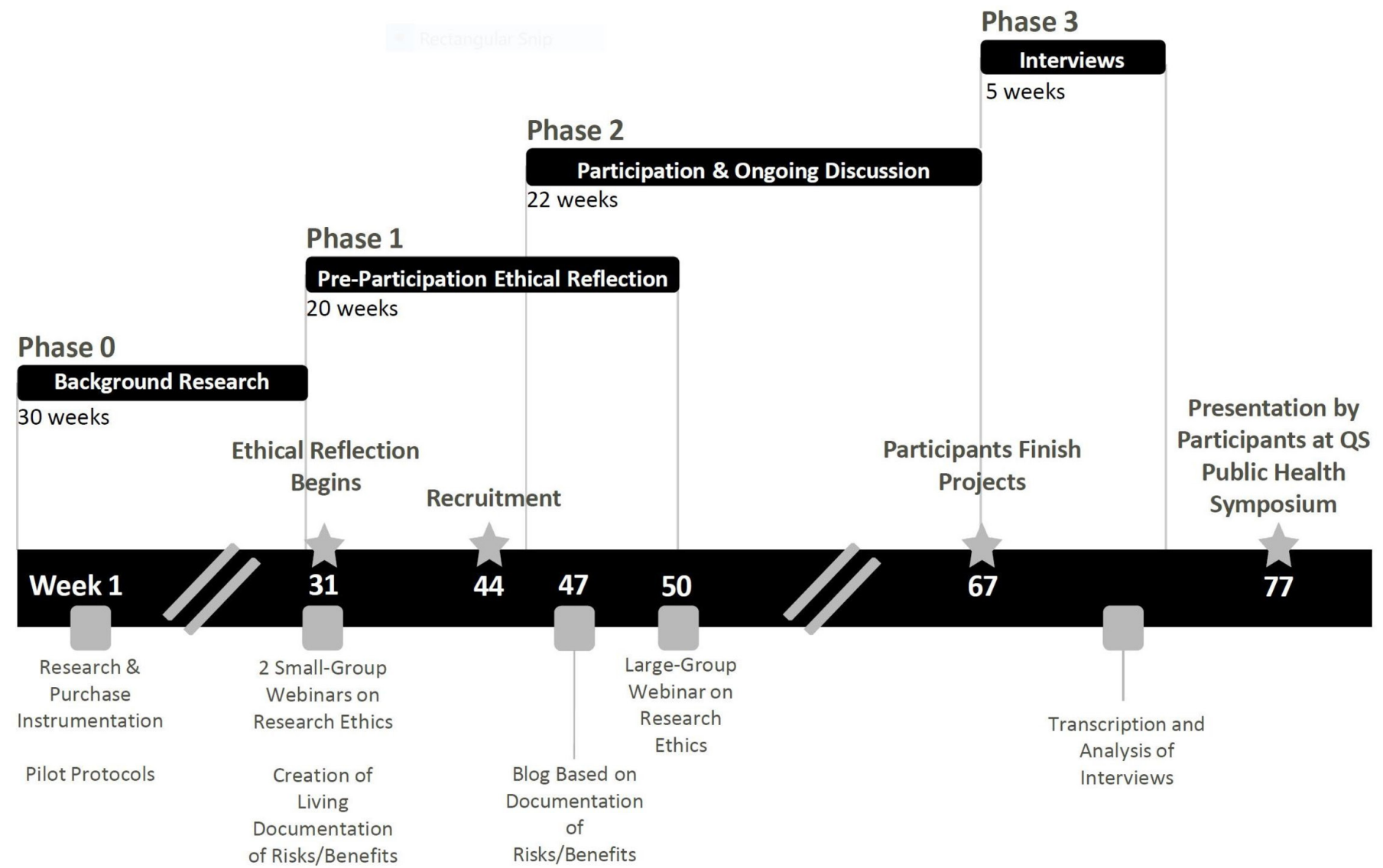

Figure 2 Timeline of Ethical Reflection in the Blood Testers PLR. Phase Zero: Participant-organisers prepared for the project by gathering supplies and piloting protocols and identifying a research ethicist. Phase One: The research ethicist, who was also a participant, led a webinar/brainstorming session on research ethics, focusing on informed consent. Documentation of this discussion was shared in the common project Google drive. Recruitment was held at a Quantified Self Global Conference, followed by an online summary of potential risks and benefits of participation. A large group webinar then shared the material of the first ethical reflection meetings with the full group of participants. This phase overlaps slightly with Phase Two, as some participants joined later than others. Phase Two: Participants kept ongoing communications with one another and participantorganisers while conducting personal experiments and data analysis. Experiment planning meetings/check-ins often included 'updates' to assessment of risks and benefits. Phase Three: Following project completion, participants were interviewed about their experience in the project. Projects were shared at the QS Public Health Symposium. PLR, participant-led research; QS, Quantified Self.

\section{Phase three}

Group communication to enable ongoing ethical reflection

During the semi-structured interviews, participants were asked about their preferences for receiving study information. As noted, several methods were used to share information and promote discussion, in order to ensure that all participants engaged in reflection on risks and benefits of participation. Direct one-on-one communications with the participant-organisers was preferred by the majority (57\%) of participants, as evidenced by the following comments: "I learnt a ton of background [in one-on-one meetings]" (P07) and "I really valued getting to ask direct questions." (P11). These one-on-one meetings incorporated discussion of potential risks and benefits into the construction of the individual research protocols, which participants said helped put the project in context. For example, P17 said: "The conversation was really good because I was more engaged with the idea of the experiment... Planning an experiment was deep work that was hard to do by myself and another person to bounce ideas off of was valuable. Instead of feeling like I just wanted to do an experiment and turn something in, I felt that my question was very interesting and I'm doing something new. It felt transparent and comfortable."

Others (35\%) engaged most during the webinars due to a preference for listening to a group's conversation, stating: "Having a group chat let me see other types of questions people had, I think it helped me get an understanding of the process (of the study)" (P16) and "Because of the type of learner I am, the webinar was more helpful because I could listen and follow along" (P09). The practice of thinking alongside other participants appeared to help some to compare and contrast their assumptions with others, thereby reflecting more critically on the PLR process. For the remaining $8 \%$, webinar recordings and written material were an absolute necessity. One participant (P01) opted to watch recorded webinars and post comments for the group, saying that "I was very busy with 
Table 1 Participant-generated risks and risk mitigation strategies

Risk Risk Mitigation

Engagement with ethical issues of participation was perceived Our challenge is to test if collaborative discussion of risks and as difficult, which could limit engagement. benefits will be more enjoyable and engaging.

Participants could learn something unpleasant (eg, results that Participants were made aware of this risk in the initial project require medical attention). discussion, before taking any lipid tests.

Frequent testing can cause some people anxiety.

Participants could be disappointed by learning the actual bounds of uncertainty of the data, even if these bounds are comparable to that of professional tests.
After some discussion, and polling of participants, we agreed that this risk is minimal in our group.

This topic was discussed at length in the beginning of the project and was also considered a benefit. Consumers often do not realise the extent to which data from at-home testing can be uncertain.

Reputation risk to participant-organisers if ethical concerns are not well understood.

Reputation risk to participant-organisers if training on how to use the test system is not effective.

\section{Participants could feel peer-pressure to carry out an experiment.}

Reputation risk to all project participants if data-quality is questionable.

Conflict of interest concern by participants regarding funding.

Demands on participants' time.

Minor pain and bruising.

Almost negligible risk of infection.

Risk of being penalised in the future based on data being read by others and associated with a sanction by insurance companies.

Quantified Self as a movement puts itself at risk by stumbling across legal and/or social liabilities.
Participant-organisers convened all participants to engage in discussion of risks and benefits.

Participants were thoroughly trained, and training materials and expertise were made available for the entire duration of the project.

Participants were encouraged to only carry out testing that was personally interesting and productive.

Participants were incentivised to collect good data because they conducted personally-relevant experiments.

Goals and funding were clearly stated to all before joining the project, and funders did not view the manuscript or advise on project content.

There was no minimal required time commitment. Our goal was to be as supportive as possible and to understand reasons for halted projects as they arrived.

Participants were trained with techniques to minimise discomfort. Participants chose how frequently to sample and

Participants were given sterile supplies and trained to use equipment safely.

All participants could keep their data private and offline. Data were removed from group-spreadsheet post-project unless participant expressed interest in keeping the data public. multiple opportunities were provided for participants to reflect. could stop at any point.

Transparency was maintained about risks and benefits, and working and caring (for a relative), but the webinars were the most informative, along with our written correspondence." Most participants moved fluidly between different types of communication; and discussion of risks and benefits naturally came up in conversation as a part of experimental planning sessions. All participants reported that ethical reflection was an important component of the project.

\section{Factors influencing informed consent}

Additionally, several participants mentioned that familiarity with the other members of the group contributed to their positive assessment of risks in relation to benefits. One participant (P05) said: "It gave me comfort walking into a study knowing that people that I knew were participating as well. It gave me comfort in what I was doing was useful, because I trust these people... If you were to take any sort of subject or any test and say,
' $\mathrm{X}, \mathrm{Y}$ and $\mathrm{Z}$ are all involved in this, would you consider joining?' The answer is that I'm probably biased towards joining because they are part of it. I know those people, and I know that they are very rational and calculated thinkers..."

While this interaction appears strongly positive, it also sets the stage for the possibility of peer-pressure or coercion in PLR. That being said, almost all of our cohort said that they would have felt comfortable halting their participation at any time. Two individuals reported 'self-pressure' during their experiments, reflecting: "It's not in any way the kind of pressure that has been put on by the group, but it is more responsibilities I have taken on for myself." (P11) or "It was my own pressure. I said I would do it." (P08). One participant (P03), brought up that it can be very challenging to avoid the possibility of coercion, thereby giving inherently low-risk PLR an 
Box 1 Participant-generated benefits of participation

\section{Benefit}

- Sharing the method of small group, collaborative self-discovery. Uncovering challenges therein is necessary for revising the process of Participant-Led Research.

- Proposing a new method for more engaging ethical review in Participant-Led Research.

- Greater community ability and motivation to validate new self-tracking tools before use.

- Educating ourselves, to the best of our ability, about the current literature in cholesterol and triglyceride research.

- Learning the extent to which individuals' lipids vary throughout the day as measured.

- Learning the extent to which a single measurement at the doctor's office is representative of one's 'regular' lipid levels.

- Increased ability to engage with one's physician in a conversation about the health-relevance of one's cholesterol and triglyceride levels.

- Increased ability to conduct an experiment, and empowerment to interrogate future personal questions using scientific tools.

- Access to costly blood-testing equipment, and the data generated by it.

- Encouragement from respected fellow participants.

- Access to advice from individuals experienced in experimental design.

- Access to help with data analysis.

- Opportunity to share learnings at a Quantified Self symposium or conference.

advantage: "One can say 'yes, it's ok, I chose to do this (experiment)', but that might be irrelevant. There is a history of people like physicians getting patients to make choices against their own interests..." Although this is a highly self-motivated cohort, there is no doubt that peer pressure could play a role in participants taking experimentation further than if they were on their own. For this reason, PLR that minimises potential risk of harm (eg, collection of wearable data) may be most appropriate while standards for PLR governance are developed.

\section{Benefits of participation - participant learning}

The key benefit expressed by participants was the assistance they received from one another in forming and interrogating their own research questions. Even individuals well-versed in data analysis sometimes struggled with defining precise experimental questions, and individuals with a background in medicine or biology were not often familiar with statistical analysis. A common outcome was that once data were plotted, with the aid of another participant, a discussion between the two yielded the most valuable insights of the project. Participants even expressed pride in their experimental outcomes, one saying "I was going around telling people that I collected 24 hours of hourly cholesterol readings, which hadn't been published before. And now I've done it... I feel like a pioneer!" (P17). In answer to a question about what would aid them in future personal experiments, participants mentioned a number of features of the Blood Testers PLR. These included help with forming research questions and protocols, statistical analysis and data visualisation. A bonus for this PLR was the creation of two short and accessible educational videos developed to explain physiological functions and patterns in the measured lipid outputs. ${ }^{39}$ Finally, all participants expressed interest in joining future PLR. See box 1 for a complete list of participant-generated benefits.

\section{Prospective consent and governance principles for participant-led research}

Nine themes emerged from discussions and interviews relating to informed consent in and governance of PLR. As this PLR was driven by people with different backgrounds asking personal questions, we found that ethical reflection needed to be ongoing and tailored to the individual. For this reason, prospective governance principles were drafted rather than codified rules. Many of the themes were expressed over the course of our PLR as an ongoing informed consent. The process, fostered via frequent communication, helped to reinforce trust among participants and organisers. ${ }^{43} 44$

1. Transparency: All relevant information about the project should be actively shared among participants and participant-organisers, including the source of research funding, equipment selection, data management protocols, risks and benefits and conflicts of interest.

2. Access to Expertise: Participant-led research (PLR) requires access to experts (eg, in experimental design, data analysis, research ethics) so that participants can rigorously carry out single-subject experiments. ${ }^{45}$

3. Data Access \& Control: The participant has the right and ability to manage their own data, and has the final say in what they collect about themselves.

4. Right to Withdraw: Participants have a right to reduce or withdraw their participation at any time.

5. Relevance: PLR addresses questions of relevance to the participants.

6. Beneficence: The participant actively reflects on the balance of benefits and risks of participation and freely choose whether to participate.

7. Responsibility: PLR requires that the participant actively consider the potential benefits and harms of the project to both themselves and others. The responsibility to stay informed is an ongoing process, not a onetime decision.

8. Flexibility: Ethical reflection in PLR should be tailored to individual needs and to the specific context, rather than be handled with 'one size fits all' rules. The needs of an individual are dynamic, and a lack of rigidity can reinforce trust between participants and organisers. ${ }^{464}$

9. Inclusivity: If a prospective participant is willing and able to uphold these principles, they are welcome to participate. 


\section{DISCUSSION}

In this PLR, a global cohort of self-trackers collaboratively identified risks, risk mitigation strategies and benefits of participation in a study of blood lipids. Participants and participant-organisers mainly identified risks associated with data aggregation and identifiability and proposed individual data management, ownership and control as risk mitigation strategies. Participant benefits centred on personal learning, access to data and diverse experimental expertise. Prospective principles were created to capture essential ethical components of the project. These principles may aid the development of governance and informed consent practices in future PLR, but leave an important question: how can PLR grow as a rigorous and ethical research practice before official governance standards are established? Further acknowledgement of the differences between PLR and traditional research, flexibility in addressing 'unknown unknowns' and commitment to crafting examples of low-risk PLR may be useful next steps.

All participants expressed interest in joining future PLR, yet we lack formal guidelines to inform ethical review for PLR not bound by the regulations protecting human research participants. For instance, in the US, these regulations apply specifically to research funded by the Department of Health and Human Services. ${ }^{35}$ This means that PLR organisers not bound by this mandate must decide whether or not to obtain review by an IRB or REC. It is important to note that the IRBs and RECs were designed for research led by professional researchers affiliated with organisations that receive government funding for biomedical and behavioural research studies involving human participants. This traditional paradigm of ethical review is obviously very different from a collaboratively-led international cohort of individuals, who may lack academic research training or exposure to research ethics, and professional researchers..$^{28} 48$ As such, IRB/ REC involvement may promote decisions specific to data ownership, data management and informed consent that directly conflict with the aims of research that is explicitly participant-led. For instance, IRBs often require that a Principal Investigator take complete responsibility for and ownership of study-generated data, which may oppose participants' expectation to own the data they collect about themselves. ${ }^{49}$ Together, the challenges of systematising ethical review, and the lack of clear precedents for divisions of leadership and ownership have led many to conclude that current ethical review guidelines must be adapted or substituted to suit participant-led initiatives. ${ }^{12}$

Recognising that PLR is a rapidly evolving form of investigation, integrating ethical review requires a commitment to addressing challenges in the unknown future. In projects directed by a group of researchers from within an academic institution, study risks and benefits are conveyed to participants by researchers. By contrast, in PLR, participants and participant-organisers seek to uncover project risks and benefits collaboratively. The very concept of risk and benefit is altered when experimental questions are determined by participants rather than by a Principal Investigator. ${ }^{50}$ For example, PLR participants may alter their course of investigation at any point (see principle 8 ). This allows the risk to benefit calculation made by the participant at the study outset to be dynamically adjusted during the study period (eg, if the individual's experimental question evolves in a way that changes their risk and benefit evaluation). However, this also means that it is not possible to anticipate every experiment to be conducted prior to the start of the study, to determine whether or not the participant understands the risks and benefits of those experiments and to ensure that the participant consents to carry them out. As noted in our proposed principles, this permission to dynamically re-evaluate risks and benefits is central to participant control.

Although formal ethical guidelines for non-governmentally funded PLR are yet to be put in place, this does not exempt PLR from ethical review in principle. This ethical review in PLR requires a common stake among all participants. This common stake means that all who take part in the project share an investment in the conduct and outcomes of the research. This stake even extends to those in traditional research conditions, in which greater attention to the participant experience stands to benefit not just the participant, but the ultimate quality of the research in terms of improved data annotation, participant retention. ${ }^{84}$ While best practices continue to be developed for specific use in higher-risk projects, low-risk, observational PLR may not need to wait for governmental guidelines to formalise its methods and contribute its findings to the scientific literature. Our experience suggests that encouraging ethical reflection among a small group while asking research questions that can be answered using low-risk procedures can safely generate participant benefits.

\section{LIMITATIONS}

As is often the case with new research methodologies, our learning is biased by our narrow context, intentional minimal-risk design and unique community of selftrackers. Larger, more diverse cohorts and other distinctive communities may find discussion based ethical-review less applicable to their context. Additionally, this project and writing of this manuscript took place prior to and during the adoption of changing ethical regulations across national borders (ie, the General Data Protection Regulation). We chose to limit our introduction largely to ethical regulatory frameworks in the US, acknowledging that the UK and EU regulations are relatively similar in content and implementation practices.

\section{CONCLUSIONS}

PLR is an emerging form of investigation in which responsibility is shared more equally between participants and participant-organisers. The PLR described in this paper is 
novel in two ways. First, it is the first PLR, to the authors' knowledge, in which all participants formed unique research questions to explore and collected and managed all data individually. Second, the cohort engaged in ethical reflection before, during and on conclusion of the project and used documentation of these discussions to create guiding principles for future PLR. This PLR retained $88 \%$ participation through its conclusion and $100 \%$ satisfaction among finishing participants. We conclude that low-risk PLR involving single-subject study in a small group may be conducted responsibly and ethically by incorporating an ethical reflection process at onset and throughout the study duration. It is our hope that the principles generated during this PLR may encourage discussion and development of ethical PLR practices.

Acknowledgements We would like to thank all participants in Blood Testers for their excellent work in shaping this PLR. We would also like to thank Dr. Sunita Vohra and Dr. Martijn de Groot for their careful reading and commentary on the manuscript.

Contributors AG led the project, which is the case under analysis in this manuscript, conducted all interviews, contributed to data analysis with $\mathrm{CN}$, and led writing and editing of the manuscript. GW contributed to conception and organisation of the project and to writing and editing of the manuscript. CN contributed to the conception and guidance of the ethical reflection process, data analysis with $A G$, and writing and editing of the manuscript.

Funding Funding for the project was provided by a grant from Amgen Inc. to Quantified Self Labs. The company did not contribute to the research question(s), methods, analysis or interpretation. Amgen Inc. did not have access to data obtained via this project and has not had the opportunity to review this paper prior to its submission for peer-reviewed publication.

Competing interests None declared.

Patient consent for publication Not required.

Provenance and peer review Not commissioned; externally peer reviewed.

Data sharing statement As interview transcripts contain personally identifying information that cannot easily be removed while preserving the content of the interview (e.g., discussion of individuals' health condition in the context of their experiments, discussion of the death of a family member's impact on trying to complete a project, descriptions of other participants in the context of their projects), transcripts will not be shared.

Open access This is an open access article distributed in accordance with the Creative Commons Attribution Non Commercial (CC BY-NC 4.0) license, which permits others to distribute, remix, adapt, build upon this work non-commercially, and license their derivative works on different terms, provided the original work is properly cited, appropriate credit is given, any changes made indicated, and the use is non-commercial. See: http://creativecommons.org/licenses/by-nc/4.0/.

\section{REFERENCES}

1. Vayena E, Brownsword R, Edwards SJ, et al. Research led by participants: a new social contract for a new kind of research. J Med Ethics 2016;42:216-9.

2. Mikesell L, Bromley E, Khodyakov D. Ethical community-engaged research: a literature review. Am J Public Health 2013;103:e7-14.

3. Bonney R, Shirk JL, Phillips TB, et al. Citizen science. Next steps for citizen science. Science 2014;343:1436-7.

4. Swan M. Crowdsourced health research studies: an important emerging complement to clinical trials in the public health research ecosystem. J Med Internet Res 2012;14:e46.

5. Pettibone L, Vohland K, Ziegler D. Understanding the (inter) disciplinary and institutional diversity of citizen science: a survey of current practice in Germany and Austria. PLoS One 2017;12:e0178778.

6. Nebeker C, López-Arenas A. Building Research Integrity and Capacity (BRIC): an educational initiative to increase research literacy among community health workers and promotores. J Microbiol Biol Educ 2016;17:41-5.

7. Wilson Center. The Rise of the New Bio-Citizen [Internet]. 2018 https://www.wilsoncenter.org/article/the-rise-the-new-bio-citizen (cited 20 Jun 2018).

8. Cox SM, McDonald M. Ethics is for human subjects too: participant perspectives on responsibility in health research. Soc Sci Med 2013;98:224-31.

9. Banks S, Armstrong A, Carter K, et al. Everyday ethics in community-based participatory research. Contemp Soc Sci 2013;8:263-77.

10. Fleurence $R$, Whicher $D$, Dunham $K$, et al. The patient-centered outcomes research institute's role in advancing methods for patientcentered outcomes research. Med Care 2015;53:2-8.

11. Jones FM, Allen C, Arteta C, et al. Time-lapse imagery and volunteer classifications from the Zooniverse Penguin Watch project. Sci Data 2018;5:180124

12. Swanson A, Kosmala M, Lintott C, et al. A generalized approach for producing, quantifying, and validating citizen science data from wildlife images. Conserv Biol 2016;30:520-31.

13. Citizen science alliance [Internet]. https://www.citizensciencealliance. org/

14. Bonney R, Cooper C, Ballard H. The theory and practice of citizen science: launching a new journal. Citizen Science: Theory and Practice 2016;1:1.

15. Follett R, Strezov V. An analysis of citizen science based research: usage and publication patterns. PLoS One 2015;10:e0143687.

16. Gura T. Citizen science: amateur experts. Nature 2013;496:259-61.

17. Cohn JP. Citizen science: can volunteers do real research? Bioscience 2008;58:192-7.

18. Hand E. Citizen science: people power. Nature 2010;466:685-7.

19. Vayena E, Tasioulas J. Adapting standards: ethical oversight of participant-led health research. PLoS Med 2013;10:e1001402.

20. Wolf G, Ramirez E. Quantified self/public health symposium, 2014.

21. Wicks P, Vaughan TE, Massagli MP, et al. Accelerated clinical discovery using self-reported patient data collected online and a patient-matching algorithm. Nat Biotechnol 2011;29:411-4.

22. Lewis D. Setting expectations for successful artificial pancreas/ hybrid closed loop/automated insulin delivery adoption. J Diabetes Sci Technol 2018;12:533-4.

23. Godlee F. Towards the patient revolution. BMJ 2014;348:g1209.

24. Doerr M, Maguire Truong A, Bot BM, et al. Formative evaluation of participant experience with mobile econsent in the app-mediated parkinson mpower study: a mixed methods study. JMIR Mhealth Uhealth 2017;5:e14.

25. Grady C, Cummings SR, Rowbotham MC. Informed Consent. Drazen JM, Harrington DP, McMurray JJV, Ware JH, Woodcock J, editors. N Engl J Med 2017;376:856-67.

26. Rothstein MA, Wilbanks JT, Brothers KB. Citizen science on your smartphone: an ELSI research agenda. J Law Med Ethics 2015;43:897-903.

27. Sugarman J. Examining provisions related to consent in the revised common rule. Am J Bioeth 2017;17:22-6.

28. Bloss $\mathrm{C}$, Nebeker $\mathrm{C}$, Bietz $\mathrm{M}$, et al. Reimagining human research protections for 21 st century science. J Med Internet Res 2016;18:e329.

29. Thorogood A, Bobe J, Prainsack B, et al. APPLaUD: access for patients and participants to individual level uninterpreted genomic data. Hum Genomics 2018:12:7.

30. Weissman JS, Campbell EG, Cohen IG, et al. IRB oversight of patient-centered outcomes research: a national survey of IRB Chairpersons. J Empir Res Hum Res Ethics 2018;13:421-31.

31. Vayena E, Tasioulas J. The ethics of participant-led biomedical research. Nat Biotechnol 2013;31:786-7.

32. U.S. Department of Health and Human Services. 45 CFR 46 protection of human subjects, 2009.

33. Bouesseau M-C, Coleman C, Kass N, et al. Standards and operational guidance for ethics review of health-related research with human participants. 2011 http://apps.who.int/iris/bitstream/handle/ 10665/44783/9789241502948 eng.pdf;jsessionid=5E488F141667 C3CEA6FED5BE49301ED4? sequence $=1$

34. Bouesseau M-C, Coleman C, Kass N, et al. Standards and Operational Guidance for Ethics Review of Health-Related Research with Human Participants: World Health Organ, 2011.

35. Department of Health, Education, and WelfareNational Commission for the Protection of Human Subjects of Biomedical and Behavioral Research. The Belmont Report. Ethical principles and guidelines for the protection of human subjects of research. J Am Coll Dent 2014;81:4-13.

36. An Introduction to Lipids on Vimeo [Internet]. https://vimeo.com/ 237116970 (cited 10 Dec 2018). 
37. Biological Rhythms on Vimeo [Internet]. https://vimeo.com/ 239682398 (cited 10 Dec 2018)

38. Aberdeen T, Yin RK. Case study research: Design and methods (4th Ed.). Thousand Oaks, CA: Sage. Can J Action Res 2013;14:69-71.

39. Creswell JW. Research design: qualitative, quantitative, and mixed methods approaches: SAGE Publications, 2009:297. p.

40. Gerring J. What is a case study and what is it good for? Am Polit Sci Rev 2004;98:341-54.

41. Patton MQ. Qualitative research \& evaluation methods : integrating theory and practice:806.

42. Grant A, Wolf G. Free-Living Humans Cross Cardiovascular Disease Risk Categories due to Biological Rhythms in Cholesterol and Triglycerides. J Circadian Rhythms (in revision).

43. Kerasidou A. Trust me, I'm a researcher!: the role of trust in biomedical research. Med Health Care Philos 2017;20:43-50.

44. Guillemin M, Gillam L, Barnard E, et al. "We're checking them out": Indigenous and non-Indigenous research participants' accounts of deciding to be involved in research. Int J Equity Health 2016;15:8.

45. Choe EK, Lee NB, Lee B, et al. Understanding quantified-selfers' practices in collecting and exploring personal data. Proceedings of the 32nd annual ACM conference on Human factors in computing systems, 2014:1143-52.

46. McDonald M, Townsend A, Cox SM, et al. Trust in health research relationships: accounts of human subjects. J Empir Res Hum Res Ethics 2008:3:35-47.

47. Guillemin M, Barnard E, Allen A, et al. Do research participants trust researchers or their institution? J Empir Res Hum Res Ethics 2018;13:285-94.

48. Nebeker C, Harlow J, Espinoza Giacinto R, et al. Ethical and regulatory challenges of research using pervasive sensing and other emerging technologies: IRB perspectives. AJOB Empir Bioeth 2017;8:266-76.

49. Gliklich RE, Dreyer NA, Leavy MB. Principles of registry ethics, data ownership, and privacy [Internet]: Agency for Healthcare Research and Quality, 2014.

50. Townsend A, Taylor K, Cox S. Conceptions of risk regarding a chronic illness survey: perspectives of participants, researchers, and ethics review board members. IRB 2014:36:13-20. 